\title{
A Study of Asphaltene Content of Indonesian Heavy Oil
}

\author{
Astra Agus Pramana ${ }^{1}$, S. Rachmat ${ }^{1}$, D. Abdassah ${ }^{1} \&$ M. Abdullah ${ }^{2}$ \\ ${ }^{1}$ Petroleum Engineering Department, Bandung Institute of Technology, Bandung, Indonesia \\ ${ }^{2}$ Physics Department, Bandung Institute of Technology, Indonesia \\ Correspondence: Astra Agus Pramana, Petroleum Engineering Department, Bandung Institute of Technology, Jl. \\ Ganesha 10, Bandung, Indonesia. E-mail: agus.prama@gmail.com
}

Received: March 15, 2012

Accepted: March 29, 2012 Online Published: May 1, 2012

doi:10.5539/mas.v6n5p64

URL: http://dx.doi.org/10.5539/mas.v6n5p64

\begin{abstract}
Oil production is highly depend on its viscosity which varies with temperature and pressure. Chemically, the viscosity had tight relationship with asphaltene content. The higher the asphaltene content as in the heavy oil, the higher the viscosity is and the more difficult to be produced. A brief review of Saturates, Aromatics, Resins and Asphaltenes (SARA) fractions of oil and how they are extracted and analysed followed by how asphaltene related with viscosity then its theoretical models and its precipitation mechanism, is given. The paper then expose the viscosity vs temperature behavior of Indonesian heavy oil and relates with its SARA measurement.
\end{abstract}

Keywords: asphaltene, heavy oil, SARA analysis

\section{Introduction}

Oil is sometimes characterized by its physical properties such as viscosity or density (API gravity) or by destillation for its light chemical compounds. But for heavy oil, SARA separation or fractionation is commonly applied which gives maltenes (saturates, aromatics, resins) and asphaltenes. Although it doesn't deal with the gases and varies depend on its solvents (see Figure 1), SARA analysis- first done in 1972 by Jewell- is the simplest way in comparing oils for upstream and downstream purposes (Jewell, 1972). The following is a brief summary of each fraction but emphasized on asphaltenes, since it is believed to be tightly related with the high viscosity of heavy oil.

Saturates or aliphatics are non polar fraction, without double bonds with branched and straight-chain alkanes (wax/parafins: C20-C30) and cyclo-alkanes (naphtenes). Saturates are lightest fraction and therefore their content usually decreases with increasing molecular weight fractions. Aromatics are benzenes and their structural derivates mostly contain alkyl chains and cyclo-alkane-rings. They are classified as mono-, di-, and tri- aromatics which refer through the number of their aromatic rings in the molecule. Aromatics are more polarizable than saturates. Resins are polar molecules mostly contain heteroatoms such as nitrogen, oxygen or sulphur. Resins have higher $\mathrm{H} / \mathrm{C}$ ratio $(1.2-1.7)$ than asphaltene $(0.9-1.2)$ but smaller molecular weight $(<1000 \mathrm{~g} / \mathrm{mole})$. Naphtenic acid, for example, is a resin.

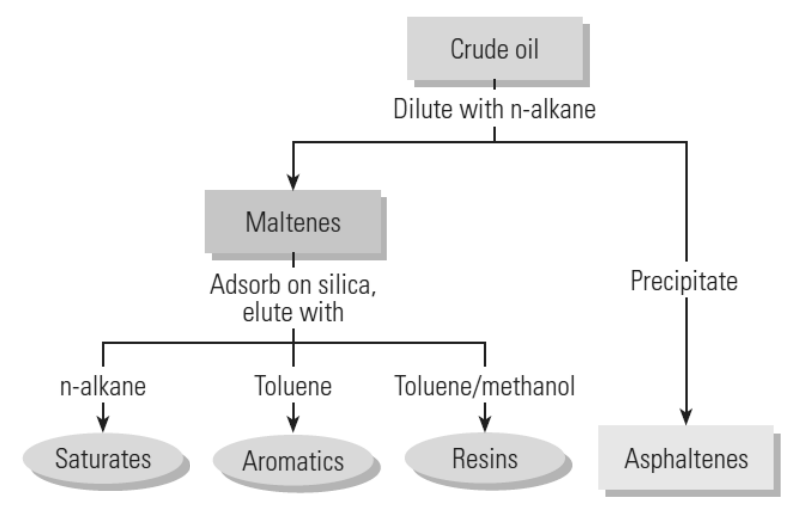

Figure 1. The SARA separation of oil (Akbarzadeh K. et al., 2007) 
Asphaltene is known to be a fraction of oil (as depicted in the Figure 2 below) which is not a unique pure compound, that is (Rastegari, 2003; Petrophysics group, 2009; Akbarzadeh, 2007):

1) Insoluble in n-alkanes (n-pentane or n-heptane) at a dilution ratio of 40 parts alkane to 1 part crude oil.

2) Soluble in toluene.

3) Dark coloured, friable, density of $1.2 \mathrm{~g} / \mathrm{cm}^{3}$, powdery, most polarized and heaviest

4) Infusible (no clear melting point but decomposed when heated)

5) Not a chemical class but has complex molecular structures of highly aromatic cores, long chain aliphatic groups,

6) Contain heteroatoms (nitrogen, sulphur, oxygen, and heavy metals such as vanadium and nickel)

7) Carbons and heteroatoms are bound in ring-like

If the asphaltene was extracted from crude oil with propane (lower alkanes: n-C5) it would be sticky, more liquid-like, brown colour as in part (a) of Figure 2. While with heptanes (n-C7), it is black coloured and not sticky. Another thing is that the extracted asphaltene from crude oil is about twice the molecular weight than that extracted from coal (Petrophysics group, 2009).

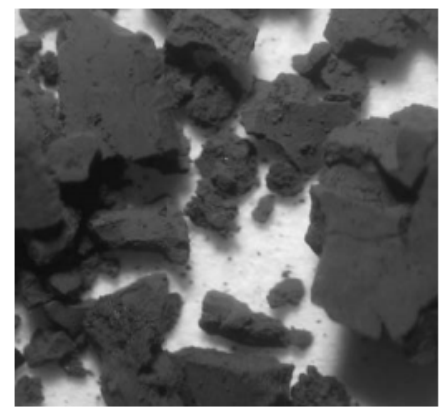

(a) $\mathrm{n}-\mathrm{C}_{5}$ asphaltenes

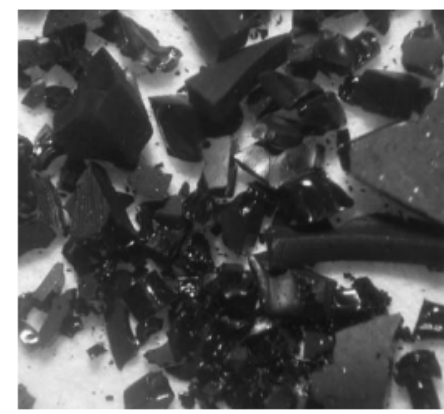

(b) $\mathrm{n}-\mathrm{C}_{7}$ asphaltenes

Figure 2. The asphaltene extraction taken from different solvents a) from $\mathrm{n}-\mathrm{C} 5 \mathrm{~b}$ ) from $\mathrm{n}-\mathrm{C} 7$ (Petrophysics group, 2009)

The standard SARA measurement was given by ASTM D2007-93 and ASTM D4124-91 which are difficult, time consuming and need large amount of sample. Better methods are using high pressure liquid chromatography (HPLC) and thin layer chromatography combined with flame ionization detection (TLC-FID). The latter is more popular since it is fast and uses small amount of sample of less than $100 \mathrm{ml}$ (Fan, 2002). Though some reported the discrepancy and inconsistency of TLC FID method for SARA analysis, mostly due to the aging of its chromarod, the calibration and standardization recipes had to be done to get clean peaks of the fractions (Masson, 2001; Bharaty, 1994).

Elemental composition of asphaltene is described by Rastegari as: $\mathrm{H} / \mathrm{C}$ ratio about $0.5 \%, 0.3-4.9 \%$ oxygen content, $0.3-10.9 \%$ sulphur content. From infrared and NMR spectroscopy, asphaltene structure consists of aromatic rings with short aliphatic chains. Their molar mass range from 600 to $300,000 \mathrm{~g} / \mathrm{mol}$ and also vary with the measurement techniques (VPO-Vapor Pressure Osmometry or GPC-Gel Permeation Chromatography) (Rastegari, 2003).

\section{Heavy Oil Viscosity Related to Its Asphaltene and Resin Content}

Viscosity is the most important physical property of the heavy oil which controls its mobility. Low viscosity will make the oil has high mobility and therefore, easier to be produced. Several papers had discussed the heavy oil viscosity, its dependence on temperature and pressure, and also its relationship to asphaltene content. The following are brief summaries of the related works.

A review of calculation methods for hydrocarbon viscosity was done by Mehrotra et al. which is separated into semi-theoretical methods and empirical methods. In the semi-theoretical methods, the reference fluid or a characterization parameter is needed to match other properties such as boiling point or specific gravity. Meanwhile, in the empirical methods, generalized parameters are regressed and used for fitting to the viscosity model. However, most of these methods are still requiring viscosity data or interaction parameters (Mehrotra, 1996).

Experimental approach is done by analyzing SARA concentrations from 7 set of heavy oil as depicted in Figure 3. It is found that summation of resin and asphaltene concentrations is linear to the viscosities of those sets and 
follow power law for their shear modulus (Hinkle, 2008). Though done only on few set of samples, the idea has been brought for not to only focusing on the asphaltene but also to the other heavy-molecular weight resins.
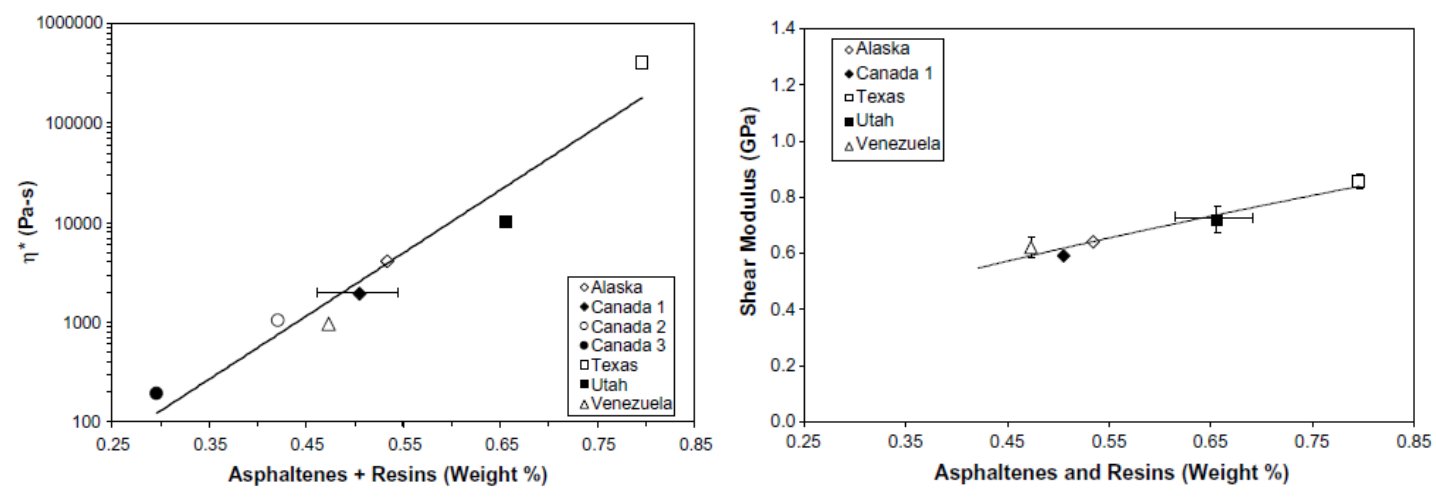

Figure 3. Arrhenius type viscosity and Shear modulus vs the content of Asphaltene+Resin (Hinkle, 2008)

With many more data, Evdokimov made a breakthrough by explaining the inconsistent hyphotesis of viscosity of dead crude oil as a function of asphaltene's content as in Figure 4. This inconsistency is mentioned as multi-peak correlation which is explained as the cause due to phase boundaries of asphaltene nanocolloids. A "minimum line" lies exponentially, below which none viscosity value is found for the related asphaltene content (Evdokimov, 2009).

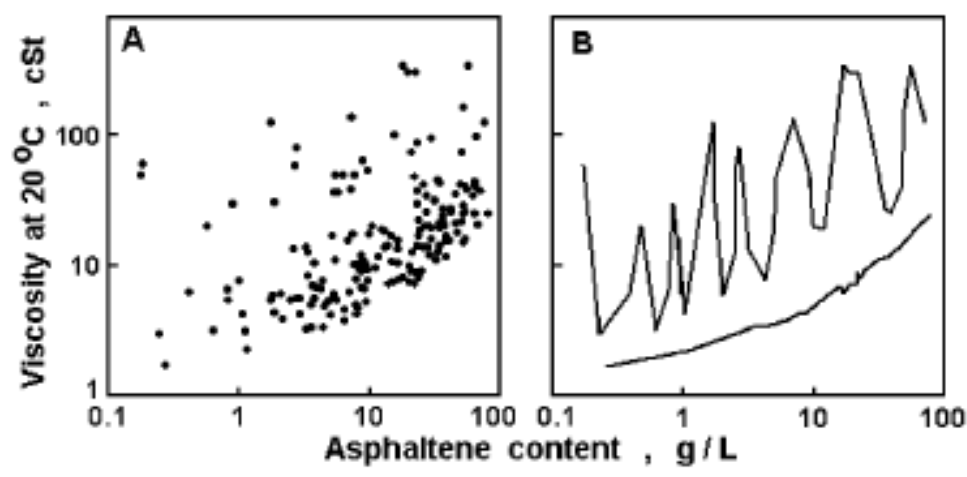

Figure 4. Viscosity vs Asphaltene content (Evdokimov, 2009)

The above result is actually a mere prove of a hypothesis brought by Argilier et al. which stated that the origin of heavy oil viscosities are from entanglement of solvated asphaltene particles. These particles are stabilized by resin molecules. Above a constant $C^{*}$, named as the critical concentration, the asphaltenes' particles overlapp and change dramatically the viscosity behavior. This overlapping is caused by the lowering asphaltene's effects done by the resins. This can be interpreted that resin had lost its ability to stabilize the asphaltene particles. Furthermore, at low temperature, the heavy oil has a shear thinning behavior and no yield stress while at high temperature, the absence of wax crytallization made it behaves like Newtonian fluid (Argilier, 2002).

Initially, the abovementioned $C^{*}$ constant was proposed by Pierre et al. using Small Angle X-ray Scattering (SAXS) spectroscopy, above which the asphaltenes are no longer independent particles but overlapped. Resins, according to Pierre et al., were modeled by nonylphenol and can reduce the asphaltenes influence on viscosity. The influence is explained as, firstly, the stabilizing agent which covering the asphaltenes' surfaces and, secondly, the dissociating agent which diminishing both: the size and the molecular mass of asphaltenes. In fact, it was shown that a small addition of alcohol in aromatic solvent could reduce this association (Pierre, 2004). The idea of 'peptizing' resin as a stabilizing agent, or a surfactant in the 'emulsion' of asphaltene and other fractions of oil was critically mentioned by Buckley as need more examinations. Mullins also opposed the idea by collecting some facts that resin has less charge and only small fraction of nanoaggregate (from centrifugation) which is not enough to stabilize asphaltene. In addition, ultrafiltration technique also shown that only small fraction of resin participates in asphaltene aggregate formation (Mullins, 2010). More discussion about this is put at the next 
subtitle as part of the asphaltene modeling.

Again, the value of $\mathrm{C}^{*}$ can be related to the maximum concentration mentioned by Sheu et al. that is $13 \%$, below which the asphaltene influence on viscosity is strong up to $0.1 \%$, another minimum limit which is not mentioned by others. The reason is explained by two-fluid model where longer-range interaction potential affecting a smaller maximum packing volume fraction (Sheu, 1994). As an additional information, the range of asphaltene content can reach upto $60 \%$, while the resin-to-asphaltene volume ratios vary from less than $1: 1$ in oils where asphaltenes are precipitated rapidly, to $1: 1$ until $20: 1$ in oils contain stabled-asphaltene (Islam, 1994).

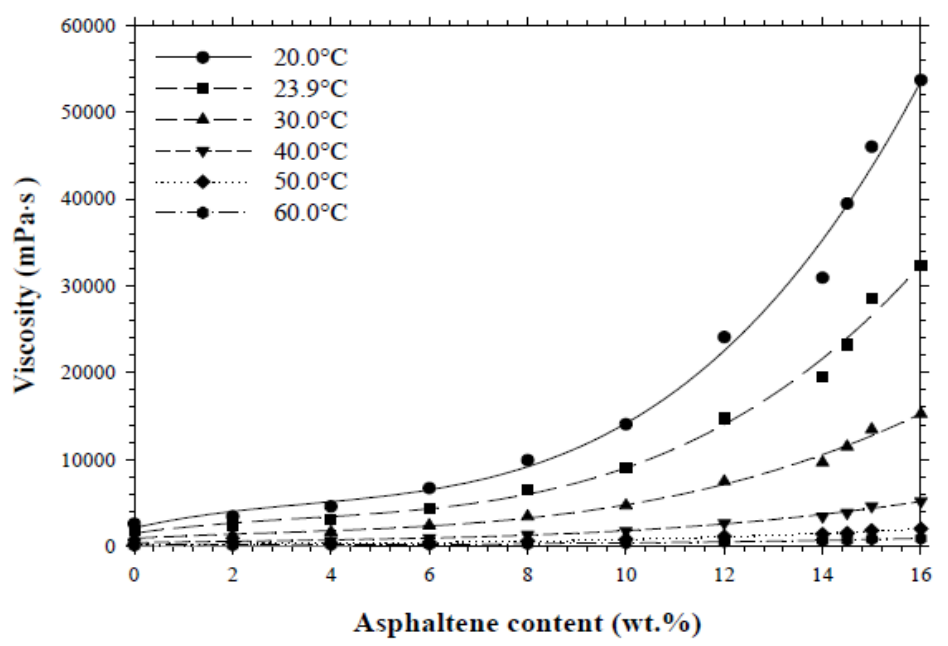

Figure 5. Viscosity vs asphaltene content and equilibrium propane pressure of a reconstituted heavy oil sample

(Luo, 2005)

Instead of finding many types of heavy oil with different asphaltene contents, Luo et al. found a short cut by reconstituting it. By adding varying asphaltene content to the deasphalted heavy oil, many samples can be obtained and their viscosities can be measured separately as seen in Figure 5. To check the validity of this method, the deasphalted heavy oil having initially $14.5 \%$ asphaltene was added $14.5 \%$ asphaltene which is found to mimick the viscosity of the initial heavy oil. As the wt $\%$ of added asphaltene increased, the viscosity of the reconstituted heavy oil is seen to slightly increase at lower temperature but more quadratic/logarithmic behavior is seen at higher temperatures. Meanwhile, a sharp viscosity drop (below $5000 \mathrm{mPa}$-s) is seen if those heavy oil exposed to solvent with at least $200 \mathrm{kPa}$ equilibrium propane pressure (Luo, 2005). It would be interesting then if the resin can also be extracted out and similarly varied with varying asphaltene content in which the Hinkle's hypotheses could be confirmed.

\section{Asphaltene Models}

Viscosity, precipitation, and other physical and chemical properties of asphaltene can be representatively explained through a model. The currently proposed models can be divided into four groups: solubility model, colloidal model, solid model, and association equation-of-state (EOS) model (Akbarzadeh, 2007) which are briefly summarized as follows:

\subsection{Solubility Model or Thermodynamic Model}

This model considers asphaltenes as macromolecules in linear or branches held by van der Waals forces with a solvent of resin, and dissolve in oil. This concept was initially proposed by Hirscberg et al. in which the resins are treated as an integrated part of the solvent medium and the asphaltene monomers and their aggregates are in thermodynamic equilibrium (Aske, 2002). Also, asphaltene precipitation depends on thermodynamic conditions such as pressure, temperature, and its composition. Therefore, this process is a thermodynamically reversible one (Hirschberg, 1984), though it is found not to be consistent in some observed behavior (Islam, 1994).

\subsection{Colloidal Model}

Colloidal models have their basis in statistical thermodynamics and colloidal science. The first such model assumed asphaltenes exist in the oil as solid particles in colloidal suspension stabilized by resins adsorbed on their surfaces. In this model, the vapor-liquid equilibrium calculated using an EOS that establishes the composition of the liquid phase from which asphaltene may flocculate. Asphaltene-precipitation measurements 
at one set of conditions are interpreted to give a critical chemical potential for resins, which is subsequently used to predict asphaltene precipitation at other conditions (Leontaritis \& Mansoori, 1987).

The colloidal theory considers asphaltenes to be solid particles of $3 \mathrm{~nm}$ size, suspended colloidally in the crude oil, consists of a stack of 6 to 10 molecules and surrounded by resin molecules. Each particle has one or more aromatic sheets of asphaltene monomers with adsorbed resins acting as surfactants to stabilize the colloidal suspension. These molecules are held together with pi-bonds, hydrogen bonds, and electron donor-acceptor bonds. When resins desorb from asphaltenes, the asphaltene aggregate size increase and start precipitating. The main reason is that asphaltene is insoluble in the resin-free oil fraction which makes the precipitation or deposition is an irreversible process. Asphaltene's degree of aromaticity and the proportion of heteroatoms contribute to its dispersive behavior. This view was supported by the appearance of disk-shaped particles, sometimes called micelles, of $8 \mathrm{~nm}$ diameter dispersed in asphaltene-toluene mixtures seen by SAXS and SANS or by electrokinetic effects. This theory is also known as stabilisation model where resins act as stabilizing agent to suspend the aggregation (Rastegari, 2003; Aske, 2002; Islam, 1994).

\subsection{Solid Model}

In this model, the precipitating asphaltene is treated as a solid-single component of the fluid and cubic EOS is used for phase modeling. Besides requiring many empirical parameters, this model is also need tuning for experimental data matching. One of this model divided the heavy crude oil components into precipitating (such as asphaltene) and nonprecipitating (Akbarzadeh, 2007).

\subsection{Modified regular Solution Model or Association Equation-of-State (EOS) Model}

Most of the simulation softwares use EOS model which initially was quite far from the reality. Therefore, a modified one, sometimes called by adding the word "association" in front of its name, was initiated by Akbarzadeh et al. This model makes four main assumptions:

- Asphaltene molecules exist mainly as monomers in the bulk crude oil and as aggregates in an associated state in the precipitation phase;

- Asphaltene association leads to asphaltene precipitation;

- The asphaltene precipitation process is thermodynamically reversible;

- The asphaltene-precipitation phase is a pseudo -liquid phase.

This model combines terms describing the chemical and physical effects of association of asphaltene molecules. It requires composition, molecular weight, molecular size and interaction energy of each component (Akbarzadeh, 2007).

\section{Asphaltene's Precipitation}

Based on the above models, the causes of precipitation, its mechanism, and even precipitation envelope which only based on the varying pressure and temperature, can be drawn. Several facts on destabilizing forces that cause asphaltene precipitation are collected by M.R. Islam and briefly summarized as follow (Islam, 1994):

- Miscible flooding which destabilizes the mixture of asphaltene-crude oil and lowers the solutions's carbon-to-hydrogen ratio and had bad effect especially at condition near the bubble point.

- $\mathrm{pH}$ shift because of $\mathrm{CO}_{2}$ injection (which also changes oil composition) or occured naturally or by organic acids produced by bacteria.

- Well stimulation such as acidizing, because it changes the chemical equilibria in the near wellbore zone.

- Pressure drop which usually accompanied by temperature change and affect resins' and maltenes' solubility, creating paraffin and asphaltene precipitation.

Meanwhile, the precipitation mechanism of asphaltene related to its size is described in Figure 6 and summarized as follows (Akbarzadeh, 2007):

a) At concentrations above $10^{-4}$ mass fraction, in toluene, a true solution of asphaltene will form nanoaggregates that dispersed as nanocolloid.

b) The stable suspension will then form clusters as the concentration reaches approximately $5 \mathrm{~g} / \mathrm{L}$, or mass fraction of $5 \cdot 10^{-3}$.

c) It is then starts to flocculate, forming clumps then precipitate beyond $10^{-2}$ mass fraction. However, asphaltenes in Athabasca bitumen can remain in a stable "solution" at very high concentration (about 18\%) without precipitating. 


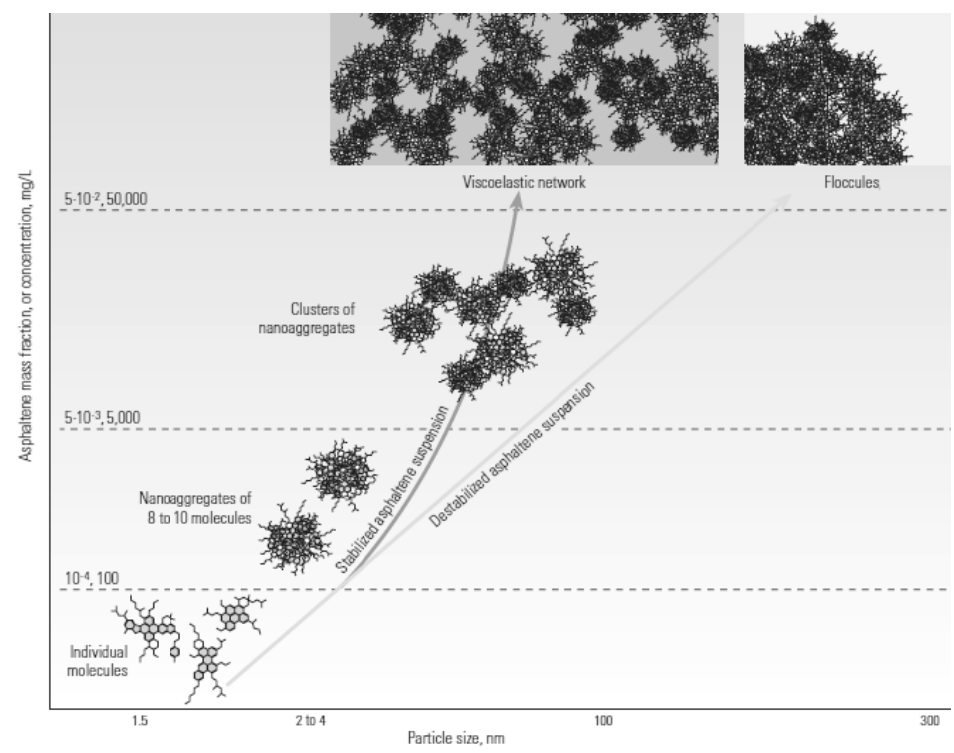

Figure 6. Relationship between asphaltene's mass fraction and the particle size of its precipitate aggregates

(Akbarzadeh K. et al., 2007)

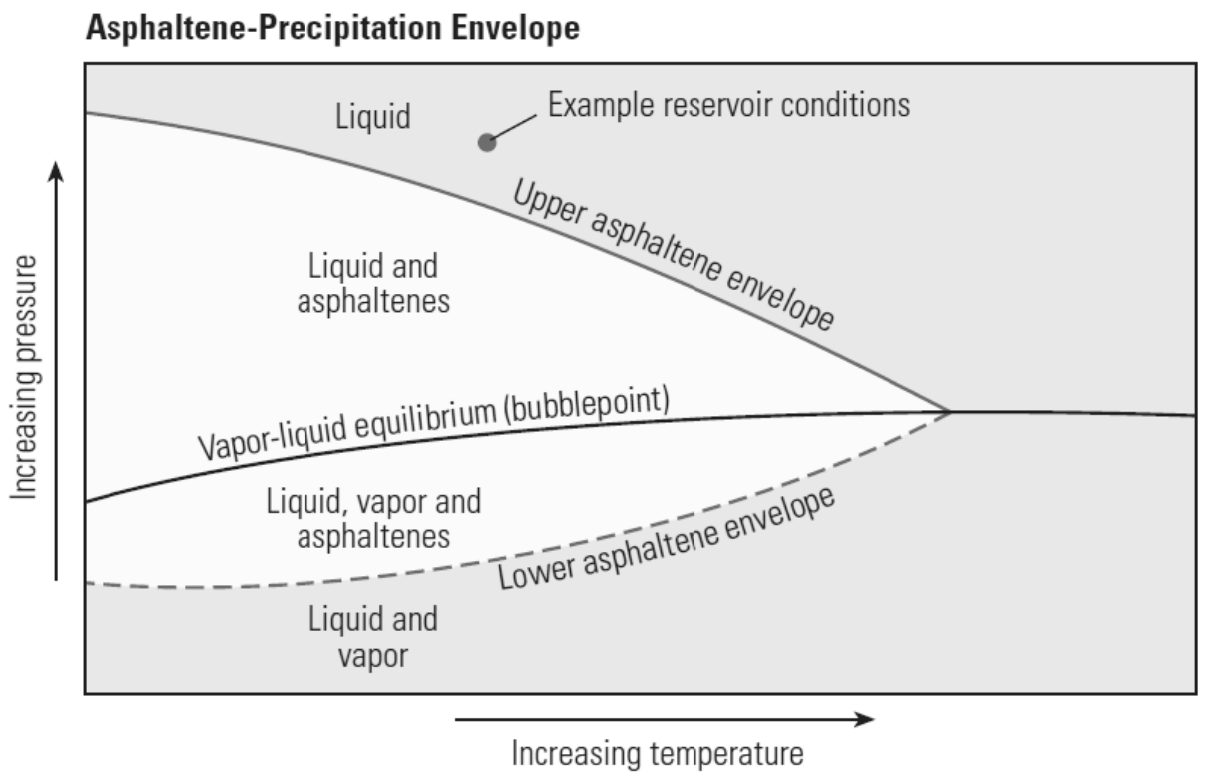

Figure 7. Asphaltene's precipitation envelope (APE) which explains its precipitation mechanism related to P-T variation (Akbarzadeh K. et al., 2007)

Knowing the causes and the mechanism of asphaltene's precipitation, an envelope model is then drawn in Figure 7 to describe how the changes in pressure and temperature affecting the precipitation.

The asphaltene precipitation envelope (APE) is the white coloured area and has upper boundary (solid red line) and lower boundary (dashed red line). In the reservoir conditions, crude oils exist at higher than the bubblepoint pressure, called undersaturated oils, which have high gas content but low asphaltene content. During production, pressure decreases to bypass the higher APE boundary (onset pressure), and the "dissolved" asphaltenes start to precipitate.

As the pressure decreases, the amount of precipitated asphaltene increases. It is then reaches a maximum at the bubblepoint pressure (solid black line). As the pressure decreases more below the bubblepoint pressure, oil becomes denser because the dissolved gas is removed. As the pressure is continually decreased, the APE's lower boundary is bypassed starting the re-dissolution of asphaltenes (Akbarzadeh, 2007). 


\section{Indonesian Heavy Oil}

The temperature dependency of viscosity of Indonesian heavy oil was measured with Fann viscometer and given in Figure 8. At $15.6^{\circ} \mathrm{C}$, the viscosity is $340 \mathrm{cP}$ which is in the lower range of heavy oil category (100 to $1000 \mathrm{cP}$ ) and its API gravity is 16.9 (heavy oil category is 10 to 22). A laboratory result using TLC-FID has been done on this heavy oil and described in Figure 9 to find its SARA content as summarized in Table 1.

The SARA graph is free of noises and needs more time to characterize the saturates compared to that of asphaltene. Higher peak of resin and asphaltene are because of their higher content and easier characterized by TLC-FID.

Table 1. SARA Content of Indonesian heavy oil

\begin{tabular}{cc}
\hline Content & Percentage \\
\hline Saturate & $31.6 \%$ \\
Aromatics & $17.7 \%$ \\
Resin & $16.2 \%$ \\
Asphaltene & $34.5 \%$ \\
\hline
\end{tabular}

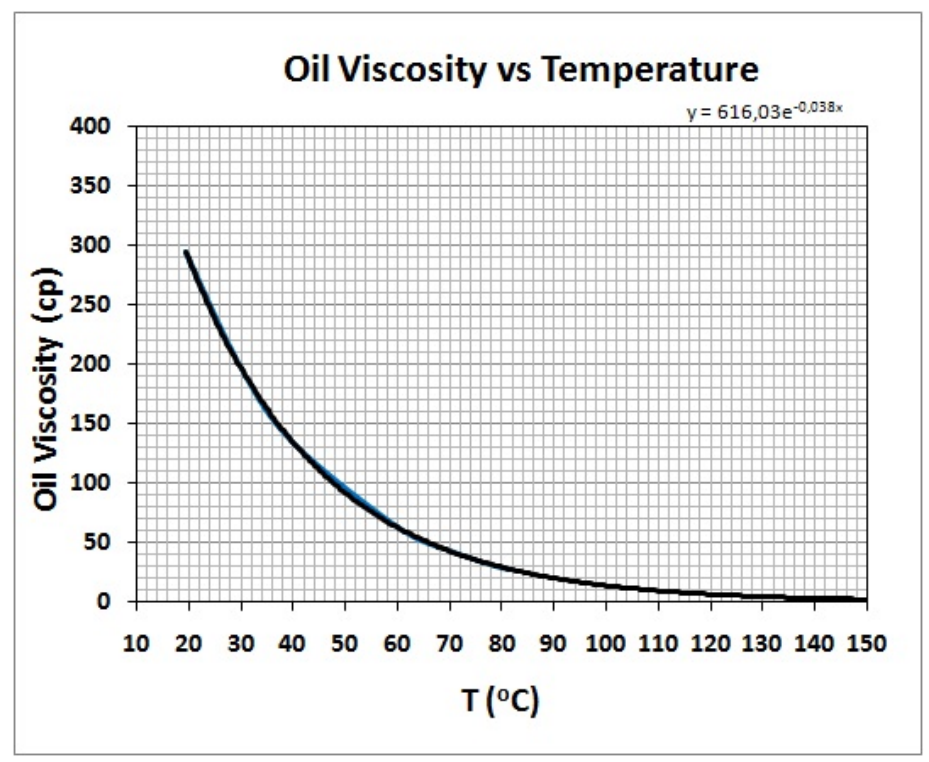

Figure 8. Viscosity variation at different temperatures for Indonesian heavy oil 


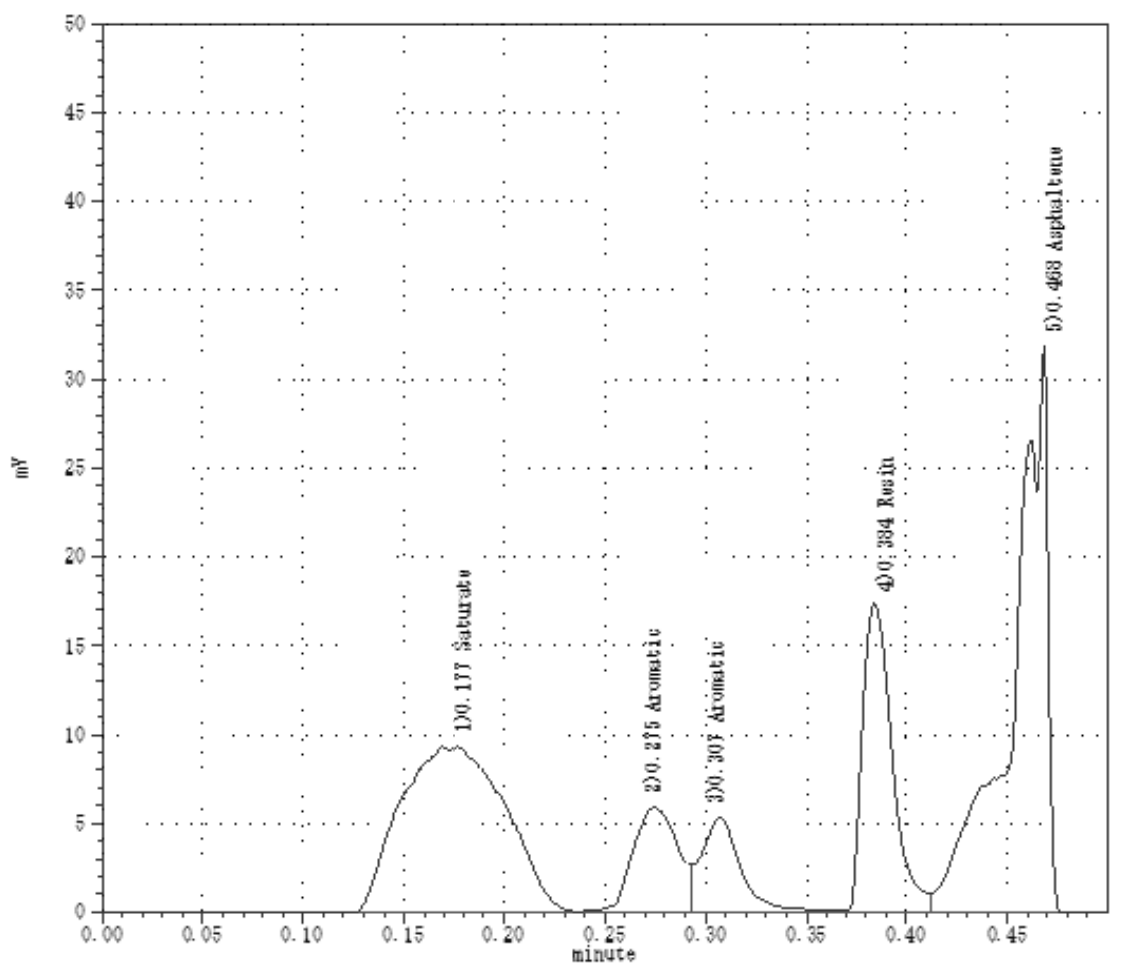

Figure 9. The SARA content of Indonesian heavy oil

Compared to Evdokimov's result (Figure 4), the relationship of asphaltene content with viscosity at $20 \mathrm{C}$ for this heavy oil is located at $(34.5 \%, 305 \mathrm{cSt})$, near to the plane of the second peak from the right. This result might support the many peak visco-asphaltene relationship of Evdokimov. Moreover, the high asphaltene content ensured that this oil has viscosity above Evdokimov's exponential line of minimum viscosity. Meanwhile, the sum of its asphaltene and resin contents which is $50.7 \%$ made the polar and heavier fractions more dominant which may be the cause of its high viscosity.

A comparison to Luo's and Hinkle's graphs cannot be done since Luo's experiment only dealt with the asphaltene content upto $18 \%$ while Hinkle's result using Arrhenius type viscosity which needs the value of activation energy.

\section{Conclusion}

A review of asphaltene extraction, measurement, precipitation and theoretical models had been explained. High asphaltene content and more dominant polar/heavier fractions had shown to cause higher viscosity of the Indonesian heavy oil. The viscosity is above the minimum Evdokimov's exponential line.

A check of onset temperature of upper APE boundary, bubble point and lower APE boundary is suggested so that the precipitation envelope can be drawn. By this, the viscosity measurement can be done either in the precipitated or unprecipitated experimental conditions.

\section{Acknowledgements}

The authors would like to acknowledge Bandung Institute of Technology for providing the research grant and to Prof. Kunio Yoshikawa for the JASSO Scholarship and a research stay at his Environ- mental and Energy Lab of Tokyo Institute of Technology, Japan.

\section{References}

Akbarzadeh, K., Hammami, A., Kharrat, A., Zhang, D., Allenson, S., Creek, J., ... Solbakken, T. (2007). Asphaltene, Problematic but Rich in Potential. Oilfield Review Magazine, Schlumberger, Houston. Retrieved from http://www.slb.com/ /media/Files/resources/oilfield_review/

Argilier, J. F., Coustet, C., Henaut, I., \& Institut Français du Petrole. (2002). Heavy Oil Rheology as a Function of Asphaltene and Resin Content and Temperature. SPE/PS-CIM/CHOA ITOHOS Conference. Calgary, Canada. http://dx.doi.org/10.2118/79496-MS 
Aske, N. (2002). Characterization of Crude Oil Components, Asphaltene Aggregate, and Emulsion Stability by means of Near Infrared Spectroscopy and Multivariate Analysis. Trondheim, Norway: Norway University of Science and Technology - Dissertation.

Bharaty, S., Røstum, G. A., \& Løberg, R. (1994). Calibration and standardization of Iatroscan (TLC-FID) using standards derived from crude oils. Advances in Organic Geochemistry, 22(3-5), 835-862. http://dx.doi.org/10.1016/0146-6380(94)90143-0

Evdokimov, I. (2009). Importance of Asphaltene Content in Petroleum II - Multi-Peak Viscosity Correlations. Petroleum Science and Technology, 27. http://dx.doi.org/10.1080/10916460902937018

Fan, T. G., Buckley, J. S. (2002). Rapid and Accurate SARA Analaysis of Medium Gravity Crude Oils. Energy and Fuels, 16, 1571-1575. http://dx.doi.org/10.1021/ef0201228

Hinkle, A., Shinb, E. J., Matthew, W. L., Herringb, A. M., \& Batzlea, M. (2008). Correlating the Chemical and Physical Properties of a set of Heavy Oils from Around the World. Fuel, 80, 3065-3070. http://dx.doi.org/10.1016/j.fuel.2008.04.018

Hirschberg, A., de Jong, L. N. J., Meijers, J. M., \& Schipper, B. A. (1984, June). Influence of Temperature and Pressure on Asphaltene Flocculation. SPE Journal, 24(3), 283-293.

Islam, M. (1994). Role of Asphaltene on Oil Recovery and Mathematical Modeling of Asphaltene Properties. Development in Petroleum Science, 40, 249-253. http://dx.doi.org/10.1016/S0376-7361(09)70258-4

Jewell, D. M., Weber, J. H., Bunger, J. W., Plancher, H., \& Latham, D. R. (1972). Ion-exchange, coordination, and adsorption chromatographic separation of heavy-end petroleum distillates. Anaytical Chemistry, 44, 1391-1395. http://dx.doi.org/10.1021/ac60316a003

Leontaritis, K., \& Mansoori, G. (1987). Asphaltene Flocculation During Oil Production and Processing, a Thermodynamic Colloidal Model. SPE International Symposium on Oilfield Chemistry. San Antonio. http://dx.doi.org/10.2118/16258-MS

Luo, P. (2005). Effects of Asphaltene Content and Solvent Concentration on Heavy Oil Viscosity. SPE/PS-CIM/CHOA ITOHOS Conference, Calgary, Canada. http://dx.doi.org/10.2118/97778-MS

Masson, J. (2001). Dynamics of Bitumen Fractions by TLC-FID. Energy \& Fuels, 15, 955-960. http://dx.doi.org/10.1021/ef0100247

Mehrotra, A. (1996). A Review of Practical Calculation Methods for the Viscosity of Liquid Hydrocarbon and their Mixtures. Fluid Phase Equilibria, 117, 344-355. http://dx.doi.org/10.1016/0378-3812(95)02971-0

Mullins, O. (2010). The Modified Yen Model. Energy Fuels, 24(4), 2179-2207.

Petrophysics group. (2009). What are Asphaltenes. Retrieved from http://baervan.nmt.edu/Petrophysics/group/intro-2-asphaltenes.pdf

Pierre, C., Barré, L., Pina, A., \& Moan, M. (2004). Composition and Heavy Oil Rheology Rhéologie des bruts lourds en relation avec leur composition. Oil \& Gas Science and Technology-Rev. IFP., 59(5), 489-501. http://dx.doi.org/10.2516/ogst:2004034

Rastegari, K. (2003). Measurement and Modeling of Asphaltene Flocculation from Athabascan Bitumen. Edmonton, Canada: Univ. of Alberta dissertation.

Sheu, E. (1994). Viscosity of Base-treated Asphaltene Solution. Fuel, 73(11), 1766-1771. http://dx.doi.org/10.1016/0016-2361(94)90165-1

Zao, B. (2005). High Temperature Behavior of Asphaltene. Edmonton, Canada: University of Alberta dissertation. 